Interfaces and Free Boundaries 15 (2013), 359-380

DOI 10.4171/IFB/306

\title{
Traveling waves from the arclength parameterization: Vortex sheets with surface tension
}

\author{
BENJAMIN AKERS \\ Department of Mathematics and Statistics, Air Force Institute of Technology, Dayton, OH, USA \\ E-mail: benjamin.akers@afit.edu \\ DAVID M. AMBrose \\ Department of Mathematics, Drexel University, Philadelphia, PA, USA \\ E-mail: ambrose@math.drexel.edu \\ J. Douglas Wright \\ Department of Mathematics, Drexel University, Philadelphia, PA, USA \\ E-mail: jdoug@math.drexel.edu
}

[Received 27 October 2012 and in revised form 21 May 2013]

\begin{abstract}
We study traveling waves for the vortex sheet with surface tension. We use the angle-arclength description of the interface rather than Cartesian coordinates, and we utilize an arclength parameterization as well. In this setting, we make a new formulation of the traveling wave ansatz. For this problem, it should be possible for traveling waves to overturn, and notably, our formulation does allow for waves with multi-valued height. We prove that there exist traveling vortex sheets with surface tension bifurcating from equilibrium. We compute these waves by means of a quasi-Newton iteration in Fourier space; we find continua of traveling waves bifurcating from equilibrium and extending to include overturning waves, for a variety of values of the mean vortex sheet strength.
\end{abstract}

2010 Mathematics Subject Classification: Primary 35C07; Secondary 76B07, 76B45.

Keywords: Arclength, vortex sheet, traveling wave, surface tension

\section{Introduction}

A fundamental question in the theory of free-surface fluid dynamics is the question of the existence of traveling waves. We study this problem for the vortex sheet with surface tension. For water waves (the case of a single fluid with vacuum above), the traveling wave problem has been extensively studied. We cannot hope to list all the papers in the literature on the topic here, but some of the fundamental contributions are [35], [39], [26], [17], [13], [37], [43], [52], [20], [21], [19], and [28].

The vortex sheet is the interface between two fluids, with a jump in tangential velocity across the interface. The fluid velocities are given by the irrotational, incompressible Euler equations. Throughout the present paper, for simplicity, we consider only the case in which the two fluids have the same density. Without surface tension, the problem is known to be ill-posed, and this illposedness can be seen to be caused by the Kelvin-Helmholtz instability. Even though the problem is ill-posed, analytic solutions (including traveling waves) exist. In the presence of surface tension, the Kelvin-Helmholtz instability is ameliorated, with growth in the linearization only for sufficiently small wavenumbers (or, depending on the parameters, there may be no growth at all).

(c) European Mathematical Society 2013 
The surface tension force appears in the formulation of the vortex sheet problem through the Laplace-Young jump condition for the pressure: $[p]=\tau \kappa$, where the brackets indicate the jump across the free surface, $p$ is the pressure, $\tau$ is the constant coefficient of surface tension, and $\kappa$ is the curvature of the free surface. Historically, this caused trouble for both the analysis and the computing of the initial value problem: curvature is nonlinear in terms of the Cartesian coordinates of the free surface, and involves multiple derivatives.

A breakthrough for the numerical solution of the initial value problem for interfacial flow with surface tension was made in the papers [31], [32]. In these papers, Hou, Lowengrub, and Shelley (HLS) formulate the initial value problem by using geometric variables rather than the Cartesian coordinates of the free surface. In particular, they describe the position of the free surface by using the tangent angle formed with the horizontal and the arclength element. If the curve is parameterized by the spatial parameter $\alpha$, then the curvature can be expressed as $\kappa=\theta_{\alpha} / s_{\alpha}$, where subscripts denote differentiation, $\theta$ is the tangent angle, and $s$ is the arclength (measured from, say, $\alpha=0$ ). Furthermore, the HLS formulation includes maintaining a (normalized) arclength parameterization of the curve at all times; this is enforced by means of an artificial tangential velocity. This choice of parameterization causes the curvature to become linear in terms of tangent angle, and the system of evolution equations becomes semilinear. Hou, Lowengrub, and Shelley then employ an implicitexplicit timestepping method [15], and this removes the stiffness from the problem; instead of a 3/2order CFL condition, there is only a first-order constraint. The HLS formulation has subsequently been used analytically as well, with a proof of short-time well-posedness in Sobolev spaces being established by the second author in [8].

The most common way to find traveling waves with respect to a certain variable, say $x$, is to seek functions of $x-c t$; that is, the profile of $f(x-c t)$, for any $f$, simply translates at speed $c$. For the vortex sheet with surface tension, if we seek waves at small amplitude, this would be sufficient: we could parameterize the free surface by horizontal position, $x$, and proceed from there. However, it should be possible to have overturning traveling waves. In the case of overturning waves, the free surface can no longer be parameterized by horizontal position, and we need to formulate the traveling wave ansatz in some other way. Our solution to this problem is straightforward and quite general; we require that the parameterized curve $(x(\alpha, t), y(\alpha, t))$ evolve according to $(x, y)_{t}=$ $(c, 0)$. A full formulation of the traveling wave problem, based on this equation, will be presented below, and we will then use this formulation as the basis of both an analytical and a computational study of traveling periodic vortex sheets with surface tension.

There have been several analytical studies previously of traveling waves for the vortex sheet, neglecting the effect of surface tension. Amick and Turner proved the existence of solitary interfacial waves on finite depth [14]. On infinite depth, Sun studied the symmetry and asymptotic properties of solitary waves [47]. Sun has also proved the existence of periodic traveling waves [48], [50]. The formulation used in [50] is notable in that it allows overturning waves, and it does this by means of a conformal mapping. The formulation of the present work also allows for overturning waves, and does so without recourse to complex analysis; thus, the present formulation should readily generalize to the case of three-dimensional fluids.

There have also been a number of previous computational studies for the vortex sheet without surface tension. In particular, overturning traveling waves for the vortex sheet without surface tension have been computed. Baker, Meiron, and Orszag made a formulation of the traveling wave problem for interfacial waves, but implemented the method only for waves with single-valued height [16]. (We note that one of the equations of the formulation of Baker, Meiron, and Orszag is the 
same as our equation (6) below.) Meiron and Saffman subsequently implemented the formulation of [16] to study traveling vortex sheets with multi-valued height; they additionally studied such waves with the formulation of Saffman and Yuen as well [44], [38]. Grimshaw and Pullin as well as Turner and Vanden-Broeck computed traveling vortex sheets without surface tension, making studies of the extreme behavior of the waves [27], [53].

For interfacial waves in the presence of surface tension, Sun and Shen have proved the existence of solitary waves when the fluids are of finite extent in the vertical direction [51]. In the case of infinite depth, Sun has proved decay properties of solitary interfacial waves [49]. We are not aware of any studies, either analytical or computational, of spatially periodic interfacial traveling waves accounting for surface tension in which the two fluids are of infinite depth. The closest articles in the literature of which we are aware are [25], which concerns itself mainly with approximate equations, and [33], which considers an interfacial system with two free surfaces (the lower fluid is of infinite depth, and the upper fluid is bounded above and below by free surfaces).

Using our formulation, we prove the existence of small-amplitude periodic traveling waves. The proof follows from the classical "bifurcation from a simple eigenvalue" results of Zeidler [59] or Crandall \& Rabinowitz [22]. Our solutions bifurcate from the flat equilibrium where the two fluids are shearing past one another with a velocity jump equal to a parameter $\gamma_{0}$ at the interface. We use the wave's speed $c$ as the bifurcation parameter. As it happens, the space of solutions of the linearization at candidate bifurcation points is two dimensional. By restricting our attention to solutions with a certain symmetry, the zero eigenvalue becomes simple and the CrandallRabinowitz-Zeidler theory applies. Consequently our solutions are symmetric: the profile is even, the tangent angle is odd, and the vortex sheet strength is even. Interestingly, for any choice of $\gamma_{0}$ we are able to find traveling waves.

We prove this theorem both because it is not previously in the literature, as we have described above, but also to demonstrate that our formulation of the traveling wave problem is amenable to analytical studies. The formulation is appropriate for numerical studies as well, and we use it to compute traveling periodic vortex sheets with surface tension.

The numerical solutions presented here are computed via a continuation scheme in which the equations are discretized and the resulting algebraic system is solved with a quasi-Newton iteration, similar to the method used repeatedly by Vanden-Broeck and collaborators [46], [53], [54]. Fourier collocation is used to discretize the equations, as in other works by the first author and collaborators [5], [4], [6]. The Birkhoff-Rott integral is computed in two parts: a Hilbert transform is computed using its definition in Fourier space, and the remainder is computed with an alternatingpoint trapezoidal rule, similarly to [11]. The quasi-Newton method of Broyden is then used to solve the resulting algebraic system [18]; this method is faster than the similar secant-method based scheme used by the first author in [2]. The linear solution is used as an initial guess for small amplitude, and large amplitude solutions are computed via numerical continuation.

The traveling, spatially periodic waves that we find in the present work are an example of timeperiodic vortex sheets with surface tension, although the time-periodicity is in a sense trivial. Nontraveling time-periodic vortex sheets with surface tension were computed previously by the second author and Wilkening in [11]; there, nontrivially time-periodic solutions were found bifurcating from the flat, still equilibrium. The work [11] used a numerical method which had previously been developed for the Benjamin-Ono equation by the same authors in [12] and [10]; a version of the method has also been applied for time-periodic water waves by Wilkening in [57]. For the Benjamin-Ono equation, the authors found nontrivially time-periodic solutions bifurcating not only 
from equilibrium, but also from a variety of periodic traveling waves. It would be of interest in the future to study whether the periodic traveling waves we find in the present work can similarly be used as starting points of continua of nontrivially time-periodic vortex sheets with surface tension.

This question of bifurcation to nontrivially time-periodic solutions is closely related to the question of spectral stability of these waves. Of course, determining the stability of the waves we compute is a question of independent interest, and is a worthy subject of future research. The authors intend to at least make a numerical study of stability; this has been done in other cases, for instance by Nicholls and by Akers and Nicholls for water waves in [40], [7]. We remark that even if these waves turn out to all be unstable, they may still be relevant to the dynamics of vortex sheets with surface tension - see, for example, the literature on unstable coherent structures in turbulent Couette flow [29], [56], [34].

Perhaps the most well-known traveling waves with multi-valued height are the waves of Crapper [23], [24]. These are pure capillary water waves, i.e., the density of the upper fluid can be taken to be zero, and the lower fluid has unit density. For Crapper waves, the effect of surface tension is accounted for, but the effect of gravity is neglected. These waves were shown to exist as Crapper produced an exact, closed-form solution. Subsequently, Okamoto established uniqueness of the Crapper waves (among solutions satisfying a positivity condition) [41], [42]. Another future direction of the current research will be to use the formulation of the current work to study capillarygravity waves nearby to Crapper waves; that is, we will endeavor to perturb the Crapper waves by adding gravity, and this could perhaps be done both analytically and numerically.

The plan of the paper is as follows: in Section 2, we give a description of the motion of a curve in terms of its tangent angle and arclength. In Section 3, we then give our formulation of the traveling wave ansatz; this comes via a detailed calculation in Section 3.1, or from a short calculation in Section 3.2. In Section 4, we specialize our formulation of traveling waves to the case of the vortex sheet with surface tension, and in Section 5 we use bifurcation theory to prove the existence of small amplitude, spatially periodic, symmetric traveling vortex sheets with surface tension. In Section 6, we give the results of computations of these waves, including waves with multi-valued height.

We thank the referees for a number of useful comments which helped us to improve this article.

\section{Description of a curve and its motion}

We consider a parameterized curve, given by $(x(\alpha, t), y(\alpha, t))$. The curve has unit tangent and normal vectors

$$
\hat{\mathbf{t}}=\frac{\left(x_{\alpha}, y_{\alpha}\right)}{s_{\alpha}}, \quad \hat{\mathbf{n}}=\frac{\left(-y_{\alpha}, x_{\alpha}\right)}{s_{\alpha}},
$$

where the arclength element, $s_{\alpha}$, is given by

$$
s_{\alpha}^{2}=x_{\alpha}^{2}+y_{\alpha}^{2} .
$$

The curve is considered to evolve according to some normal velocity, $U$, and some tangential velocity, $V$ :

$$
(x, y)_{t}=U \hat{\mathbf{n}}+V \hat{\mathbf{t}} .
$$

If the curve is described by its tangent angle, $\theta$, and by $s_{\alpha}$, then we use the definition

$$
\theta=\tan ^{-1}\left(\frac{y_{\alpha}}{x_{\alpha}}\right),
$$


and we can infer the evolution equations

$$
\theta_{t}=\frac{U_{\alpha}+V \theta_{\alpha}}{s_{\alpha}}, \quad s_{\alpha t}=V_{\alpha}-\theta_{\alpha} U .
$$

In the spatially periodic case, we consider solutions for which $x(\alpha+2 \pi, t)=x(\alpha, t)+M$, and $y(\alpha+2 \pi, t)=y(\alpha, t)$. The length of one period of the curve, $L$, is given by

$$
L=\int_{0}^{2 \pi} s_{\alpha} d \alpha
$$

and the time derivative of this is

$$
L_{t}=\int_{0}^{2 \pi} s_{\alpha t} d \alpha=-\int_{0}^{2 \pi} \theta_{\alpha} U d \alpha .
$$

If we take the curve to be parameterized by (normalized) arclength, then we would have $s_{\alpha}=L / 2 \pi$, and so we must have $s_{\alpha t}=L_{t} / 2 \pi$. This specifies the tangential velocity, up to a constant of integration:

$$
V_{\alpha}=\theta_{\alpha} U-\frac{L_{t}}{2 \pi}=\theta_{\alpha} U-\frac{1}{2 \pi} \int_{0}^{2 \pi} \theta_{\alpha} U d \alpha .
$$

Notice that the length $L$ can be recovered from the periodicity:

$$
M=x(\alpha+2 \pi)-x(\alpha)=\int_{0}^{2 \pi} x_{\alpha} d \alpha=\int_{0}^{2 \pi} \frac{L}{2 \pi} \cos (\theta) d \alpha .
$$

Therefore,

$$
L=\frac{2 M \pi}{\int_{0}^{2 \pi} \cos (\theta) d \alpha} .
$$

Of course, this could have been written as

$$
s_{\alpha}=\frac{M}{\int_{0}^{2 \pi} \cos (\theta) d \alpha} .
$$

\section{The traveling wave ansatz}

In this section, we formulate the traveling wave problem for a parameterized curve, using the normalized arclength parameterization. We give two versions of the calculation, one longer and one shorter.

\subsection{Detailed calculation of the traveling wave ansatz}

We now look for conditions which would guarantee that such a moving curve is a traveling wave with respect to the $x$ variable. This would mean that we could take an arclength parameterization of the curve for which

$$
x_{t}=c, \quad y_{t}=0,
$$

where $c$ is the constant speed of the traveling wave. 
From the equation $(x, y)_{t}=U \hat{\mathbf{n}}+V \hat{\mathbf{t}}$, we see that

$$
y_{t}=\frac{U x_{\alpha}+V y_{\alpha}}{s_{\alpha}} .
$$

Therefore, setting $y_{t}=0$ implies

$$
U x_{\alpha}+V y_{\alpha}=0 .
$$

Furthermore, the traveling wave ansatz implies that the length of one period of the curve is not changing, so $L_{t}=0$. The expression for the tangential velocity from the above discussion on the arclength parameterization is then the following:

$$
V_{\alpha}=\theta_{\alpha} U
$$

We see, therefore, that we have two expressions relating the normal and tangential velocities. We now solve (1) and (2) for $U$ and $V$.

We begin by remarking that since $\theta=\tan ^{-1}\left(y_{\alpha} / x_{\alpha}\right)$, we have $\tan (\theta)=y_{\alpha} / x_{\alpha}$. Combining this observation with (1), we see that

$$
U \cot (\theta)+V=0 .
$$

We differentiate this:

$$
U_{\alpha} \cot (\theta)-U \theta_{\alpha} \csc ^{2}(\theta)+V_{\alpha}=0
$$

We use (2) to substitute for $V_{\alpha}$ :

$$
U_{\alpha} \cot (\theta)-U \theta_{\alpha} \csc ^{2}(\theta)+U \theta_{\alpha}=0 .
$$

Using the identity $1-\csc ^{2}(\theta)=-\cot ^{2}(\theta)$, this becomes

$$
U_{\alpha} \cot (\theta)-U \theta_{\alpha} \cot ^{2}(\theta)=0 .
$$

Notice that this is a separable differential equation relating $U$ and $\theta$ !

We rewrite (3), separating variables:

$$
\frac{U_{\alpha}}{U}=\theta_{\alpha} \cot \theta .
$$

Integrating this, we have $\ln |U|=\ln |\sin (\theta)|+d$, for some constant $d$. Solving for $U$, we finally arrive at

$$
U=A \sin (\theta),
$$

for some constant $A$. Now that we have an expression for $U$, we seek an expression for $V$. We use the equation $V_{\alpha}=\theta_{\alpha} U$, finding now that $V_{\alpha}=A \theta_{\alpha} \sin (\theta)$. We integrate, finding $V=-A \cos (\theta)+\beta$, for some constant $\beta$. We also have the equation $U x_{\alpha}+V y_{\alpha}=0$, and we have $x_{\alpha}=s_{\alpha} \cos (\theta)$ and $y_{\alpha}=s_{\alpha} \sin (\theta)$. Taken together, these formulas imply

$$
s_{\alpha} A \sin (\theta) \cos (\theta)-s_{\alpha} A \sin (\theta) \cos (\theta)+\beta s_{\alpha} \sin (\theta)=0,
$$

and therefore (assuming the curve is not exactly flat), we see that $\beta=0$. Our formula for $V$ is then

$$
V=-A \cos (\theta) \text {. }
$$


Notice that so far, we have not used the equation $x_{t}=c$. From the equation $(x, y)_{\alpha}=U \hat{\mathbf{n}}+V \hat{\mathbf{t}}$, we see that

$$
x_{t}=\frac{-U y_{\alpha}+V x_{\alpha}}{s_{\alpha}}=c .
$$

Substituting the formulas $x_{\alpha}=s_{\alpha} \cos (\theta), y_{\alpha}=s_{\alpha} \sin (\theta)$, as well as (4) and (5), we find

$$
x_{t}=\frac{-s_{\alpha} A \sin ^{2}(\theta)-s_{\alpha} A \cos ^{2}(\theta)}{s_{\alpha}}=-A=c .
$$

So, the constant $A$ is equal to $-c$, where $c$ is the speed of the traveling wave. Our final formulas for $U$ and $V$ are

$$
\begin{gathered}
U=-c \sin (\theta), \\
V=c \cos (\theta) .
\end{gathered}
$$

Notice that what we have seen is that the equation $U=-c \sin (\theta)$ together with the arclength parameterization implies the equation for $V$.

\subsection{Short calculation of the traveling wave ansatz}

We have $U \hat{\mathbf{n}}+V \hat{\mathbf{t}}=(c, 0)$. We can write $\hat{\mathbf{t}}=(\cos (\theta), \sin (\theta))$, and $\hat{\mathbf{n}}=(-\sin (\theta), \cos (\theta))$. This implies the following system of equations:

$$
\begin{gathered}
-U \sin (\theta)+V \cos (\theta)=c, \\
U \cos (\theta)+V \sin (\theta)=0 .
\end{gathered}
$$

The solution of this system is, as before, $U=-c \sin (\theta)$ and $V=c \cos (\theta)$. Note that the arclength parameterization assumption implied that $V_{\alpha}=\theta_{\alpha} U$ and thus $U=-c \sin (\theta)$ implies $V=c \cos (\theta)$. And so we only need to use the first of these.

\subsection{Higher-order equations}

The primary equation for our traveling wave formulation is $U=-c \sin (\theta)$, and for evolution problems which are first-order in time, this would be sufficient to specify the wave. For evolutionary systems, however, we will need to supplement this. In general, we expect that taking time derivatives of the equation $U=-c \sin (\theta)$ will be sufficient for this purpose. Note that if we take the time derivative, we get

$$
U_{t}=-c \theta_{t} \cos (\theta) .
$$

Since $\theta_{t}=\left(U_{\alpha}+V \theta_{\alpha}\right) / s_{\alpha}$, we see that (6) and (7) imply $\theta_{t}=0$, and thus our second equation becomes simply

$$
U_{t}=0 \text {. }
$$

If there were a reason to have a system which is yet higher-order in time, then we would have higher time-derivatives of $U$ equal to zero as well. 


\section{Equations of motion for the vortex sheet with surface tension}

For a vortex sheet, the normal velocity, $U$, is the normal component of the Birkhoff-Rott integral (for a derivation of the Birkhoff-Rott integral from the incompressible, irrotational Euler equations, the interested reader might consult [45]). We introduce a bit of notation: we define $\Phi: \mathbb{R}^{2} \rightarrow \mathbb{C}$ to be the complexification map,

$$
\Phi(a, b)=a+i b .
$$

We also let the complex conjugate of a complex number, $w$, be denoted by $w^{*}$. Note that for $\mathbf{u}, \mathbf{v} \in$ $\mathbb{R}^{2}$ we have:

$$
\mathbf{u} \cdot \mathbf{v}=\operatorname{Re}\left[\Phi(\mathbf{u}) \Phi(\mathbf{v})^{*}\right]
$$

We denote the image of the interface under $\Phi$ by $z$ :

$$
\Phi(x(\alpha, t), y(\alpha, t))=z(\alpha, t) .
$$

Then, with the periodicity condition $z(\alpha+2 \pi)=z(\alpha)+M$, the Birkhoff-Rott integral, $\mathbf{W}$, is given by

$$
\Phi(\mathbf{W})^{*}=\frac{1}{2 i M} \mathrm{PV} \int_{0}^{2 \pi} \gamma\left(\alpha^{\prime}\right) \cot \left(\frac{\pi}{M}\left(z(\alpha)-z\left(\alpha^{\prime}\right)\right)\right) d \alpha^{\prime} .
$$

Here, $\gamma$ is the vortex sheet strength, which is related to the jump in tangential velocity across the sheet. The formula (10) can be found, for instance, in [31], [32], or [8], for specific values of $M$. To reiterate, the normal velocity is given by

$$
U=\mathbf{W} \cdot \hat{\mathbf{n}} \text {. }
$$

We remark that the formula (10) for $\mathbf{W}$ is an integral over one period of the interface. The BirkhoffRott integral is based upon the Biot-Savart integral for recovery of the velocity from the vorticity; thus, another form of the Birkhoff-Rott integral is instead an integral over the real line, since the interface (on which the vorticity is supported) is unbounded. The reduction of the Birkhoff-Rott integral on the line to (10) uses a complex analysis theorem of Mittag-Leffler [1]. While we are using complex notation for the location of the interface, the only use of complex analysis in the present work is the use of this theorem of Mittag-Leffler to arrive at (10); this is convenient, but not essential. In the case of a doubly-periodic interface in three-dimensional fluids, there is not a corresponding reduction available so that the Birkhoff-Rott integral may be expressed as the integral over a single periodic cell of the interface. Instead, the integral over the entire, unbounded interface must be used. For computational studies, the fast evaluation method of [9] for this integral could be used.

For a vortex sheet, there is another point of view we can take on the traveling wave ansatz. Namely, fluid flow away from the interface is likewise transported at the speed $c$. This implies that the vortex sheet strength satisfies $\gamma(\alpha, t)=\gamma(\alpha, 0)$. Therefore, making use of (11) and (6) the following are the equations that we want to solve:

$$
\mathbf{W} \cdot \hat{\mathbf{n}}+c \sin (\theta)=0, \quad \gamma_{t}=0 .
$$

We have already observed that $U=-c \sin (\theta)$ implies $\theta_{t}=0$; combined with the equation $\gamma_{t}=0$ from (12), this implies (8). 
All that remains in order to specify the system (12) is to give the equation for $\gamma_{t}$. This we get, for instance, from [8]:

$$
\gamma_{t}=\frac{\tau \theta_{\alpha \alpha}}{s_{\alpha}}+\left(\frac{(V-\mathbf{W} \cdot \hat{\mathbf{t}}) \gamma}{s_{\alpha}}\right)_{\alpha} .
$$

We note that there would be several more terms on the right-hand side of this equation if we allowed the two fluids to have different densities, but for simplicity, we are considering the density-matched case at present.

\subsection{The traveling wave equations}

By assumption $s_{\alpha}$ is constant. Using this and (7) converts the equation $\gamma_{t}=0$ to $\tau \theta_{\alpha \alpha}+$ $((c \cos (\theta)-\mathbf{W} \cdot \hat{\mathbf{t}}) \gamma)_{\alpha}=0$. Thus (12) is equivalent to solving the following system:

$$
\begin{gathered}
\mathbf{W} \cdot \hat{\mathbf{n}}+c \sin (\theta)=0, \\
\tau \theta_{\alpha \alpha}+((c \cos (\theta)-\mathbf{W} \cdot \hat{\mathbf{t}}) \gamma)_{\alpha}=0 .
\end{gathered}
$$

Noting that we can write the tangent and normal vectors as

$$
\Phi(\hat{\mathbf{t}})=e^{i \theta}, \quad \Phi(\hat{\mathbf{n}})=i e^{i \theta},
$$

allows us to express the normal and tangential components of $\mathbf{W}$ using (9) and (10) as

$$
\mathbf{W} \cdot \hat{\mathbf{n}}=\operatorname{Re}\left\{\frac{e^{i \theta}}{2 M} \mathrm{PV} \int_{0}^{2 \pi} \gamma\left(\alpha^{\prime}\right) \cot \left(\frac{\pi}{M}\left(z(\alpha)-z\left(\alpha^{\prime}\right)\right)\right) d \alpha^{\prime}\right\},
$$

and

$$
\mathbf{W} \cdot \hat{\mathbf{t}}=\operatorname{Re}\left\{\frac{e^{i \theta}}{2 i M} \mathrm{PV} \int_{0}^{2 \pi} \gamma\left(\alpha^{\prime}\right) \cot \left(\frac{\pi}{M}\left(z(\alpha)-z\left(\alpha^{\prime}\right)\right)\right) d \alpha^{\prime}\right\} .
$$

Setting $\gamma=\gamma_{0}=$ constant and $\theta=0$ gives a solution of these equations for any $c$. In light of this we fix $\gamma_{0}$ and let

$$
\gamma(\alpha)=\gamma_{0}+\omega(\alpha)
$$

where we demand

$$
\int_{0}^{2 \pi} \omega(\alpha) d \alpha=0
$$

That is to say, we are fixing the mean vortex strength to be $\gamma_{0}$. (Below, we will be using $c$, the wave speed, as a bifurcation parameter, but not $\gamma_{0}$.)

Substituting this into (13) and (14), along with (15) and (16) gives:

$$
\Psi_{1}(\theta, \omega ; c):=\operatorname{Re}\left\{\frac{e^{i \theta}}{2 M} \mathrm{PV} \int_{0}^{2 \pi}\left(\gamma_{0}+\omega\left(\alpha^{\prime}\right)\right) \cot \left(\frac{\pi}{M}\left(z(\alpha)-z\left(\alpha^{\prime}\right)\right)\right) d \alpha^{\prime}\right\}+c \sin (\theta)=0
$$


and

$$
\begin{aligned}
& \Psi_{2}(\theta, \omega ; c):=\tau \theta_{\alpha \alpha} \\
& +\left[\left(c \cos (\theta)-\operatorname{Re}\left\{\frac{e^{i \theta}}{2 i M} \mathrm{PV} \int_{0}^{2 \pi}\left(\gamma_{0}+\omega\left(\alpha^{\prime}\right)\right) \cot \left(\frac{\pi}{M}\left(z(\alpha)-z\left(\alpha^{\prime}\right)\right)\right) d \alpha^{\prime}\right\}\right)\left(\gamma_{0}+\omega\right)\right]_{\alpha}
\end{aligned}
$$

We will sometimes write this more succinctly as

$$
\boldsymbol{\Psi}(\mathbf{u} ; c)=0,
$$

where $\mathbf{u}:=(\theta, \omega)$ and $\boldsymbol{\Psi}:=\left(\Psi_{1}, \Psi_{2}\right)$.

\section{Small amplitude solutions via bifurcation}

We will prove the existence of nontrivial solutions of (20) by means of classical bifurcation analysis. In particular we will use this version of the Crandall-Rabinowitz-Zeidler theorem (see [60], p. 311):

THEOREM 1 Let $\mathcal{H}^{\prime}$ and $\mathcal{H}$ be Hilbert spaces, and let $u_{0} \in \mathcal{H}^{\prime}$. Let $U$ be an open neighborhood of $u_{0}$ in $\mathcal{H}^{\prime}$. Suppose that

(H1) $\psi: U \times \mathbb{R} \longrightarrow \mathcal{H}$ is $C^{2}$.

(H2) For all $c \in \mathbb{R}, \psi\left(u_{0}, c\right)=0$.

(H3) For some $c_{0}, L\left(c_{0}\right):=\psi_{u}\left(u_{0}, c_{0}\right)$ has a one dimensional kernel and has Fredholm index equal to zero.

(H4) If $e \in \mathcal{H}^{\prime}$ spans the kernel of $L\left(c_{0}\right)$ and $f \in \mathcal{H}$ spans the kernel of $L^{\dagger}\left(c_{0}\right)$, then $\left\langle f, \psi_{u c}\left(0, c_{0}\right) e\right\rangle_{\mathcal{H}} \neq 0$.

If these four conditions hold, then there exists a sequence $\left\{\left(u_{n}, c_{n}\right)\right\}_{n \in \mathbb{N}} \subset \mathcal{H}^{\prime} \times \mathbb{R}$ with

a. $\lim _{n \rightarrow \infty}\left(u_{n}, c_{n}\right)=\left(u_{0}, c_{0}\right)$.

b. $u_{n} \neq u_{0}$ for all $n \in \mathbb{N}$ and

c. $\psi\left(u_{n}, c_{n}\right)=0$.

We are able to apply this theorem more or less directly to (20), though there are a few minor wrinkles. First we need to specify what are $\mathcal{H}^{\prime}$ and $\mathcal{H}$. It happens that our analysis is greatly simplified if we restrict attention to symmetric solutions. Specifically, let

$$
\mathcal{H}^{\prime}:=H_{o}^{2} \times H_{e, 0}^{1}
$$

where

$$
H_{o}^{s}:=\left\{f \in H^{s}: f \text { is odd }\right\}
$$

and

$$
H_{e, 0}^{s}:=\left\{f \in H^{s}: f \text { is even and } \int_{0}^{2 \pi} f(a) d a=0\right\} .
$$

That is to say, we are looking for solutions for which $\theta$ is odd, $\omega$ is even and for which $\omega$ has zero average (as discussed above). Note that $H^{s}$ refers to the usual $L^{2}$-based Sobolev space of index $s$.

Next, we specify $u_{0}$ and $U$. We can see that (20) is satisfied when the interface is perfectly flat and the two fluids are shearing past one another, i.e., when

$$
\theta(\alpha) \equiv 0 \quad \text { and } \quad \omega(\alpha) \equiv 0 .
$$


In this situation, it is straightforward to check that

$$
z=\frac{M}{2 \pi} \alpha, \quad V=c, \quad s_{\alpha}=\frac{M}{2 \pi}, \quad \mathbf{W}=0, \text { and } \hat{\mathbf{t}}=(1,0) .
$$

Thus we have

$$
\boldsymbol{\Psi}(0,0 ; c)=0,
$$

for all $c \in \mathbb{R}$. That is, we have (H2), with $u_{0}=(0,0)$.

The map $\boldsymbol{\Psi}$ is not defined on all of $\mathcal{H}^{\prime}$, because of the need to avoid self-intersections of the interface. In studies of initial value problems, such as in [8] for the vortex sheet with surface tension or in [58] for the water wave without surface tension, self-intersections are avoided by enforcing a chord-arc condition. We could use a chord-arc condition here as well, or we could simply take a sufficiently small ball around $\theta=0$. For simplicity, we choose the second option; clearly, there exists $\bar{c}>0$ such that if $\|\theta\|_{H^{2}}<\bar{c}$, then the interface cannot self-intersect, and $\boldsymbol{\Psi}$ is well-defined. We then let $U$ be the corresponding subset of $\mathcal{H}^{\prime}$, i.e.,

$$
U=\left\{\theta \in H_{0}^{2}:\|\theta\|_{H^{2}}<\bar{c}\right\} \times H_{e, 0}^{1} .
$$

We have the following Lemma, which verifies that (H1) holds:

Lemma 1 The function $\boldsymbol{\Psi}(\theta, \gamma ; c)$ defined in (18), (19) and (20) is a $C^{2}$ map from $U \times \mathbb{R}$ into $H_{o}^{1} \times H_{o}^{0}$.

Proof. This is proved directly from the definition of $\boldsymbol{\Psi}$. To prove that $\boldsymbol{\Psi}$ maps functions with the symmetry of $\mathcal{H}^{\prime}$ into those with the symmetry of $\mathcal{H}$ is not short, but neither is it interesting, and so we omit it. To prove that the map is $C^{2}$ is likewise direct from the definition of $\boldsymbol{\Psi}$ and, in particular, is implied by results in [8].

Given this, we take

$$
\mathcal{H}:=H_{o}^{1} \times L_{o}^{2},
$$

where, of course, $L_{o}^{2}:=H_{o}^{0}$. We conclude that (H1) is satisfied. To check (H3) and (H4) we must compute

$$
L(c):=\left.\frac{\partial \boldsymbol{\Psi}}{\partial \mathbf{u}}\right|_{(0,0 ; c)} .
$$

We do so now.

\subsection{Linearization about a flat surface}

To linearize we set

$$
\theta=\epsilon \theta_{1} \text { and } \omega=\epsilon \omega_{1}
$$

and also

$$
z=\frac{M}{2 \pi} \alpha+\epsilon z_{1}, \quad V=c+\epsilon V_{1}, \quad s_{\alpha}=\frac{M}{2 \pi}+\epsilon \sigma_{1}, \quad \mathbf{W}=\epsilon \mathbf{W}_{1}, \text { and } \hat{\mathbf{t}}=(1,0)+\epsilon \mathbf{t}_{1} .
$$

Of course, $z_{1}, \ldots, \mathbf{t}_{1}$ are actually functions of $\theta$ and $\gamma$. We postpone more precise formulae for them for the moment. Note here that $\epsilon$ is inserted purely for convenience. We will insert all of the above into our system and keep terms of $O(\epsilon)$ only. 
Making the substitutions into (18) we get

$\operatorname{Re}\left\{\frac{e^{i \epsilon \theta_{1}}}{2 M} \mathrm{PV} \int_{0}^{2 \pi}\left(\gamma_{0}+\epsilon \omega_{1}\left(\alpha^{\prime}\right)\right) \cot \left(\frac{1}{2}\left(\alpha-\alpha^{\prime}\right)+\frac{\epsilon \pi}{M}\left(z_{1}(\alpha)-z_{1}\left(\alpha^{\prime}\right)\right)\right) d \alpha^{\prime}\right\}=-c \sin \left(\epsilon \theta_{1}\right)$.

Expanding to $O(\epsilon)$ :

$$
\begin{aligned}
\operatorname{Re}\left\{\frac{1}{2 M} \mathrm{PV} \int_{0}^{2 \pi}\right. & \left.\omega_{1}\left(\alpha^{\prime}\right) \cot \left(\frac{1}{2}\left(\alpha-\alpha^{\prime}\right)\right) d \alpha^{\prime}\right\} \\
& -\operatorname{Re}\left\{\frac{\pi}{2 M^{2}} \mathrm{PV} \int_{0}^{2 \pi} \gamma_{0}\left(z_{1}(\alpha)-z_{1}\left(\alpha^{\prime}\right)\right) \csc ^{2}\left(\frac{1}{2}\left(\alpha-\alpha^{\prime}\right)\right) d \alpha^{\prime}\right\}=-c \theta_{1} .
\end{aligned}
$$

Now, given $\theta$, one recovers $z$ from it via the relation $z_{\alpha}=\Phi(\hat{\mathbf{t}}) s_{\alpha}=e^{i \theta} s_{\alpha}$. Plugging in from (21) and (22) we have:

$$
z_{\alpha}=\left(\frac{M}{2 \pi}+\epsilon \sigma_{1}\right)\left(1+i \epsilon \theta_{1}\right)=\frac{M}{2 \pi}+\epsilon\left(\sigma_{1}+i \theta_{1}\right)+O\left(\epsilon^{2}\right) .
$$

Also, we saw in Section 2 that

$$
s_{\alpha}=\frac{M}{\int_{0}^{2 \pi} \cos \left(\theta\left(\alpha^{\prime}\right)\right) d \alpha^{\prime}} .
$$

So, using (21), we have

$$
s_{\alpha}=\frac{M}{\int_{0}^{2 \pi} 1-\epsilon^{2} \theta_{1}^{2}\left(\alpha^{\prime}\right)+O\left(\epsilon^{4}\right) d \alpha^{\prime}}=\frac{M}{2 \pi}+O\left(\epsilon^{2}\right) .
$$

This implies that $\sigma_{1}=0$. In turn, this implies that

$$
\partial_{\alpha} z_{1}=i \theta_{1},
$$

which is to say that $z_{1}$ is a purely imaginary function plus a constant. Thus

$$
\operatorname{Re}\left\{\mathrm{PV} \int_{0}^{2 \pi} \gamma_{0}\left(z_{1}(\alpha)-z_{1}\left(\alpha^{\prime}\right)\right) \csc ^{2}\left(\frac{1}{2}\left(\alpha-\alpha^{\prime}\right)\right) d \alpha^{\prime}\right\}=0
$$

since the integrand is purely imaginary. So the linearization of (18) is

$$
\frac{1}{2 M} \mathrm{PV} \int_{0}^{2 \pi} \omega_{1}\left(\alpha^{\prime}\right) \cot \left(\frac{1}{2}\left(\alpha-\alpha^{\prime}\right)\right) d \alpha^{\prime}=-c \theta_{1} .
$$

If we denote

$$
H f(\alpha):=\frac{1}{2 \pi} \int_{0}^{2 \pi} f(a) \cot \left(\frac{1}{2}(\alpha-a)\right) d a,
$$

then we can write (23) succinctly:

$$
\frac{M c}{\pi} \theta_{1}+H \omega_{1}=0
$$


$H$ is, of course, the periodic Hilbert transform; background on the periodic Hilbert transform can be found in [30]. It has the properties that $H^{2}=-$ id when applied to functions with zero mean, and $H(\beta)=0$ for any constant, $\beta$.

Now we will linearize (19). At $O(\epsilon)$, after plugging (21) and (22) in, we have

$$
\tau \partial_{\alpha}^{2} \theta_{1}+\gamma_{0}\left(V_{1}-W_{11}\right)_{\alpha}+c \partial_{\alpha} \omega_{1}=0 .
$$

We have introduced the notation $W_{11}:=\mathbf{W}_{1} \cdot(1,0)$. We must answer the question, What are $V_{1}$ and $W_{11}$ in terms of $\theta_{1}$ and $\omega_{1}$ ? We have $V=c \cos (\theta)=c \cos \left(\epsilon \theta_{1}\right)=c+O\left(\epsilon^{2}\right)$. So $V_{1}=0$.

Also, $\mathbf{W} \cdot(1,0)=\operatorname{Re}\left(\Phi(\mathbf{W})^{*}\right)$. So, we have

$$
\mathbf{W} \cdot(1,0)=\operatorname{Re}\left\{\frac{1}{2 i M} \int_{0}^{2 \pi}\left(\gamma_{0}+\epsilon \omega_{1}\right) \cot \left(\frac{1}{2}\left(\alpha-\alpha^{\prime}\right)+\frac{\epsilon \pi}{M}\left(z_{1}(\alpha)-z_{1}\left(\alpha^{\prime}\right)\right)\right) d \alpha^{\prime}\right\} .
$$

Then

$$
\begin{aligned}
\mathbf{W} \cdot(1,0)=\epsilon \operatorname{Re} & \left\{\frac{1}{2 i M} \int_{0}^{2 \pi} \omega_{1} \cot \left(\frac{1}{2}\left(\alpha-\alpha^{\prime}\right)\right) d \alpha^{\prime}\right\} \\
& -\epsilon \gamma_{0} \operatorname{Re}\left\{\frac{\pi}{2 i M^{2}} \int_{0}^{2 \pi} \csc ^{2}\left(\frac{1}{2}\left(\alpha-\alpha^{\prime}\right)\right)\left(z_{1}(\alpha)-z_{1}\left(\alpha^{\prime}\right)\right) d \alpha^{\prime}\right\}+O\left(\epsilon^{2}\right) .
\end{aligned}
$$

The first term is zero, since the integrand is real and there is prefactor of $i$. The second term can be rewritten using the following integration by parts:

$$
\begin{aligned}
\int_{0}^{2 \pi} \csc ^{2}\left(\frac{1}{2}\left(\alpha-\alpha^{\prime}\right)\right)\left(z_{1}(\alpha)-z_{1}\left(\alpha^{\prime}\right)\right) d \alpha^{\prime} & \\
=2 \int_{0}^{2 \pi} \partial_{\alpha^{\prime}}\left(\cot \left(\frac{1}{2}\left(\alpha-\alpha^{\prime}\right)\right)\right) & \left(z_{1}(\alpha)-z_{1}\left(\alpha^{\prime}\right)\right) d \alpha^{\prime} \\
& =2 \int_{0}^{2 \pi} \cot \left(\frac{1}{2}\left(\alpha-\alpha^{\prime}\right)\right) \partial_{\alpha} z_{1}\left(\alpha^{\prime}\right) d \alpha^{\prime} .
\end{aligned}
$$

Recall that we showed above that $\partial_{\alpha} z_{1}=i \theta_{1}$. So we have

$$
\mathbf{W} \cdot(1,0)=-\epsilon \gamma_{0} \operatorname{Re}\left\{\frac{\pi}{M^{2}} \int_{0}^{2 \pi} \cot \left(\frac{1}{2}\left(\alpha-\alpha^{\prime}\right)\right) \theta_{1}\left(\alpha^{\prime}\right) d \alpha^{\prime}\right\}+O\left(\epsilon^{2}\right) .
$$

Thus we have shown

$$
W_{11}=-\frac{\gamma_{0} \pi}{M^{2}} \int_{0}^{2 \pi} \cot \left(\frac{1}{2}\left(\alpha-\alpha^{\prime}\right)\right) \theta_{1}\left(\alpha^{\prime}\right) d \alpha^{\prime}=-\frac{2 \gamma_{0} \pi^{2}}{M^{2}} H \theta_{1} .
$$

We use this expression for $W_{11}$ and the fact that $V_{1}=0$ in (25) to get the linearization of (19):

$$
\tau \partial_{\alpha}^{2} \theta_{1}+\frac{2 \gamma_{0}^{2} \pi^{2}}{M^{2}} \partial_{\alpha} H \theta_{1}+c \partial_{\alpha} \omega_{1}=0
$$

This, together with (24), gives the following formula for $L(c):=\left.\frac{\partial \boldsymbol{\Psi}}{\partial \mathbf{u}}\right|_{(0,0 ; c)}$ :

$$
L(c) \mathbf{u}_{1}=\left[\begin{array}{cc}
\mu c & H \\
\tau \partial_{\alpha}^{2}+2\left(\gamma_{0} / \mu\right)^{2} \partial_{\alpha} H & c \partial_{\alpha}
\end{array}\right] \mathbf{u}_{1},
$$


where $\mathbf{u}_{1}=\left(\theta_{1}, \omega_{1}\right)$ and

$$
\mu:=M / \pi
$$

\subsection{The kernels of $L\left(c_{0}\right)$ and $L^{\dagger}\left(c_{0}\right)$}

In this section, we check (H3) in Theorem 1. The functions $\theta$ and $\gamma$ are $2 \pi$ periodic, and so it is our moral duty to represent them as Fourier series. That is, we have $\theta(\alpha)=\sum_{k \in \mathbb{Z}} \widehat{\theta}(k) e^{i k \alpha}$ and $\gamma(\alpha)=$ $\sum_{k \in \mathbb{Z}} \widehat{\gamma}(k) e^{i k \alpha}$ where $\widehat{f}(k):=\frac{1}{2 \pi} \int_{0}^{2 \pi} f(x) e^{-i k x} d x$. We have $\widehat{H f}(k)=-i \operatorname{sgn}(k) \widehat{f}(k)$. Using the Fourier representation we can represent $L(c)$ as the Fourier multiplier operator:

$$
\widehat{L}(c):=\left[\begin{array}{cc}
\mu c & -i \operatorname{sgn}(k) \\
-\tau k^{2}+2\left(\gamma_{0} / \mu\right)^{2}|k| & c i k
\end{array}\right] .
$$

(That is, $\widehat{L(c) \mathbf{u}}=\widehat{L}(c) \widehat{\mathbf{u}}$.)

To apply Theorem 1 , we must find $c_{0}$ so that $L\left(c_{0}\right)$ has a nontrivial kernel. This occurs when $\operatorname{det} \widehat{L}(c)=0$ for some $k \in \mathbb{Z}$, or equivalently, when $|k|\left(\mu c^{2}+2\left(\gamma_{0} / \mu\right)^{2}-\tau|k|\right)=0$. So either $k=0$ or

$$
c^{2}=\frac{1}{\mu}\left(\tau|k|-2\left(\frac{\gamma_{0}}{\mu}\right)^{2}\right)
$$

for some nonzero integer $k$.

Let

$$
k_{0}:=\max \left\{1,\left\lfloor\frac{2}{\tau}\left(\frac{\gamma_{0}}{\mu}\right)^{2}\right\rfloor\right\} .
$$

The first possible bifurcation value for $c$ is

$$
c_{0}^{2}:=\frac{1}{\mu}\left(\tau\left|k_{0}\right|-2\left(\frac{\gamma_{0}}{\mu}\right)^{2}\right) .
$$

A routine calculation shows that the following functions are annihilated by $L\left(c_{0}\right)$ :

$$
\mathbf{e}_{1}(\alpha):=\kappa\left[\begin{array}{c}
\sin \left(k_{0} \alpha\right) \\
-\mu c_{0} \cos \left(k_{0} \alpha\right)
\end{array}\right], \quad \mathbf{e}_{2}(\alpha):=\kappa\left[\begin{array}{c}
\cos \left(k_{0} \alpha\right) \\
\mu c_{0} \sin \left(k_{0} \alpha\right)
\end{array}\right] \quad \text { and } \quad \mathbf{e}_{3}:=\left[\begin{array}{l}
0 \\
1
\end{array}\right],
$$

where $\kappa \in \mathbb{R}$ is taken so that the functions have unit length; these form an orthonormal basis of the kernel. Note that (H3) in Theorem 1 requires the kernel to be one dimensional, and it appears that this is not the case. However, note that by our selection of $\mathcal{H}^{\prime}$ above to be functions with a certain symmetry and such that the second component has mean zero, we can eliminate two dimensions. Specifically, only $\mathbf{e}_{1}$ is in $\mathcal{H}^{\prime}$. And so we have

$$
\operatorname{ker} L\left(c_{0}\right)=\operatorname{span}\left\{\mathbf{e}_{1}\right\} .
$$

Thus we need only establish that the map has Fredholm index zero. That is to say, we need to establish the fact that coker $L\left(c_{0}\right)$ is one dimensional when $L\left(c_{0}\right)$ is viewed as a map from $\mathcal{H}^{\prime}$ to $\mathcal{H}$. 
First, calculations completely analogous to those which led to the formulae for $\mathbf{e}_{1}$ and $\mathbf{e}_{2}$ show that the following functions are annihilated by $L^{\dagger}\left(c_{0}\right)$ (the adjoint of $L\left(c_{0}\right)$ ):

$$
\mathbf{f}_{1}:=\left[\begin{array}{c}
c_{0} k_{0} \sin \left(k_{0} \alpha\right) \\
\sin \left(k_{0} \alpha\right)
\end{array}\right], \quad \mathbf{f}_{2}:=\left[\begin{array}{c}
c_{0} k_{0} \cos \left(k_{0} \alpha\right) \\
\cos \left(k_{0} \alpha\right)
\end{array}\right] \quad \text { and } \quad \mathbf{f}_{3}:=\left[\begin{array}{l}
0 \\
1
\end{array}\right] .
$$

The adjoint is a map from the dual space of $\mathcal{H}$ into the dual space of $\mathcal{H}^{\prime}$. It is straightforward to see that $\mathcal{H}$ is self-dual and thus we eliminate two directions as before. Specifically, we have:

$$
\operatorname{ker} L^{\dagger}\left(c_{0}\right)=\operatorname{span}\left\{\mathbf{f}_{1}\right\} .
$$

We introduce the decompositions $\mathcal{H}=A_{k_{0}} \oplus \widetilde{A}$ and $\mathcal{H}^{\prime}=B_{k_{0}} \oplus \widetilde{B}$; here, the subspaces $A_{k_{0}}$ and $B_{k_{0}}$ correspond to the span of the basis functions with wavenumbers $\pm k_{0}$, and $\widetilde{A}$ and $\widetilde{B}$ are the complementary subspaces. Recall that for all functions in $\mathcal{H}$ or $\mathcal{H}^{\prime}$, the mean is zero, so there are no modes corresponding to the wavenumber zero in $\widetilde{A}$ or $\widetilde{B}$. Notice that $L\left(c_{0}\right)$ maps $B_{k_{0}}$ to $A_{k_{0}}$ and $\widetilde{B}$ to $\widetilde{A}$. We define an operator $\Gamma$ as $\widehat{\Gamma \mathbf{u}}(k)=\widehat{\Gamma}(k) \widehat{\mathbf{u}}(k)$, with the symbol given by

$$
\widehat{\Gamma}(k):=\frac{1}{\mu c_{0}^{2} i k+i \operatorname{sgn}(k)\left(-\tau k^{2}+2\left(\gamma_{0} / \mu\right)^{2}|k|\right)}\left[\begin{array}{cc}
c_{0} i k & i \operatorname{sgn}(k) \\
\tau k^{2}-2\left(\gamma_{0} / \mu\right)^{2}|k| & \mu c_{0}
\end{array}\right],
$$

when $k \neq 0, \pm k_{0}$. Notice that the denominator is nonzero since we have excluded the only wavenumbers which could lead to the denominator being zero. We define the component operators $\Gamma_{i j}$, with $i$ and $j$ in $\{1,2\}$, through their symbols, as follows:

$$
\begin{aligned}
& \widehat{\Gamma}_{11}(k)=\frac{c_{0} i k}{\mu c_{0}^{2} i k+i \operatorname{sgn}(k)\left(-\tau k^{2}+2\left(\gamma_{0} / \mu\right)^{2}|k|\right)}, \\
& \widehat{\Gamma}_{12}(k)=\frac{i \operatorname{sgn}(k)}{\mu c_{0}^{2} i k+i \operatorname{sgn}(k)\left(-\tau k^{2}+2\left(\gamma_{0} / \mu\right)^{2}|k|\right)}, \\
& \widehat{\Gamma}_{21}(k)=\frac{\tau k^{2}-2\left(\gamma_{0} / \mu\right)^{2}|k|}{\mu c_{0}^{2} i k+i \operatorname{sgn}(k)\left(-\tau k^{2}+2\left(\gamma_{0} / \mu\right)^{2}|k|\right)}, \\
& \widehat{\Gamma}_{22}(k)=\frac{\mu c_{0}}{\mu c_{0}^{2} i k+i \operatorname{sgn}(k)\left(-\tau k^{2}+2\left(\gamma_{0} / \mu\right)^{2}|k|\right)}
\end{aligned}
$$

We write $\widetilde{A}=\widetilde{A}^{(1)} \times \widetilde{A}^{(2)}$ and $\widetilde{B}=\widetilde{B}^{(1)} \times \widetilde{B}^{(2)}$. That is, $\widetilde{A}^{(1)}$ is the subspace of $H_{o}^{1}$ which corresponds to the span of the basis functions in all wavenumbers except zero and $k_{0}$, and $\widetilde{A}^{(2)}$ is the similar subspace of $L_{o}^{2}$. Similarly, $\widetilde{B}^{(1)}$ is the corresponding subspace of $H_{o}^{2}$, and $\widetilde{B}^{(2)}$ is the corresponding subspace of $H_{e, 0}^{1}$.

The $\Gamma_{i j}$ are bounded linear operators between the following spaces:

$$
\begin{aligned}
& \Gamma_{11}: \widetilde{A}^{(1)} \rightarrow \widetilde{B}^{(1)}, \\
& \Gamma_{12}: \widetilde{A}^{(2)} \rightarrow \widetilde{B}^{(1)}, \\
& \Gamma_{21}: \widetilde{A}^{(1)} \rightarrow \widetilde{B}^{(2)}, \\
& \Gamma_{22}: \widetilde{A}^{(2)} \rightarrow \widetilde{B}^{(2)} .
\end{aligned}
$$

To establish this, the asymptotics of the symbols of the $\Gamma_{i j}$, as $|k| \rightarrow \infty$, are helpful. For instance, we see that $\Gamma_{11}$ is smoothing by one derivative since its symbol behaves like $k^{-1}$ for large $|k|$. 
Inspection of the other symbols reveals that $\Gamma_{12}$ is smoothing by two derivatives, $\Gamma_{21}$ is of order zero, and $\Gamma_{22}$ is smoothing by two derivatives. Also, note that the symbols of $\Gamma_{11}$ and $\Gamma_{12}$ are real, so these operators map odd functions to odd functions. The symbols of $\Gamma_{21}$ and $\Gamma_{22}$ are pure imaginary, so these operators map odd functions to even functions. Since each of the $\Gamma_{i j}$ operators is bounded, we conclude that $\Gamma$ is bounded as well.

We have shown that $\Gamma$ is a bounded map from $\widetilde{A}$ into $\widetilde{B}$. Moreover, considering the restriction to $\widetilde{B}$, we see that $\Gamma$ is the inverse of $L\left(c_{0}\right) \uparrow \widetilde{B}$. On the other hand, the range of $L\left(c_{0}\right) \uparrow B_{k_{0}}$ is closed since this subspace is two-dimensional. Therefore the range of $L\left(c_{0}\right)$ is also closed. We conclude that the dimension of the cokernel of $L\left(c_{0}\right)$ agrees with the dimension of the kernel of $L^{\dagger}\left(c_{0}\right)$. Thus we have $(\mathrm{H} 3)$.

\subsection{The final steps}

Now we are in a position to check (H4). All we need to do is show that $\left\langle\mathbf{f}_{1}, \partial_{c} L\left(c_{0}\right) \mathbf{e}_{1}\right\rangle_{\mathfrak{H}}$ is non-zero. Clearly,

$$
\partial_{c} L\left(c_{0}\right)=\left[\begin{array}{cc}
\mu & 0 \\
0 & \partial_{\alpha}
\end{array}\right]
$$

Thus

$$
\partial_{c} L\left(c_{0}\right) \mathbf{e}_{1}=\kappa\left[\begin{array}{c}
\mu \sin \left(k_{0} \alpha\right) \\
\mu c_{0} k_{0} \sin \left(k_{0} \alpha\right)
\end{array}\right] .
$$

Since $\mathcal{H}=H_{o}^{1} \times L_{o}^{2}$ we have

$$
\begin{aligned}
\left\langle\mathbf{f}_{1}, \partial_{c} L\left(c_{0}\right) \mathbf{e}_{1}\right\rangle_{\mathcal{H}} & =\left\langle c_{0} k_{0} \sin \left(k_{0} \cdot\right), \kappa \mu \sin \left(k_{0} \cdot\right)\right\rangle_{H^{1}}+\left\langle\sin \left(k_{0} \cdot\right), \kappa \mu c_{0} k_{0} \sin \left(k_{0} \cdot\right)\right\rangle_{L^{2}} \\
& =c_{0} k_{0} \mu \kappa\left(\left\|\sin \left(k_{0} \cdot\right)\right\|_{H^{1}}^{2}+\left\|\sin \left(k_{0} \cdot\right)\right\|_{L^{2}}^{2}\right) \\
& \neq 0 .
\end{aligned}
$$

Thus, we have solutions and the following is proven:

THEOREM 2 For all $\gamma_{0} \in \mathbb{R}$, there exists a non-zero sequence of functions $\left\{\left(\theta_{n}(\alpha, t), \gamma_{n}(\alpha, t)\right)\right\}_{n \in \mathbb{N}} \subset H^{2} \times H^{1}$, and a sequence of real numbers $\left\{c_{n}\right\}_{n \in \mathbb{N}}$, such that for all $n \in \mathbb{N},\left(\theta_{n}(\alpha, t), \gamma_{n}(\alpha, t)\right)$ is a nontrivial traveling wave solution with speed $c$ for the vortex sheet with surface tension. As $n \rightarrow \infty$ we have $c_{n} \rightarrow c_{0}$ and $\left(\theta_{n}, \gamma_{n}\right) \rightarrow\left(0, \gamma_{0}\right)$. Moreover, $\theta_{n}$ is always an odd function of $\alpha$ and $\gamma_{n}$ is a always an even function of $\alpha$. Lastly, $\frac{1}{2 \pi} \int_{0}^{2 \pi} \gamma_{n}(a, t) d a=\gamma_{0}$ for all $t$.

\section{Numerical results}

To complement the small-amplitude results of Section 5, we numerically compute branches of traveling waves for a variety of amplitudes. Throughout this section, we specialize to the case $M=2 \pi$. To compute solutions, we use a Fourier spectral decomposition of both the tangent angle and the vortex sheet strength

$$
\theta(\alpha, 0)=\sum_{n=-N / 2}^{N / 2} a_{n} e^{i k_{n} \alpha}, \quad \gamma(\alpha, 0)=\sum_{n=-N / 2}^{N / 2} b_{n} e^{i k_{n} \alpha} .
$$




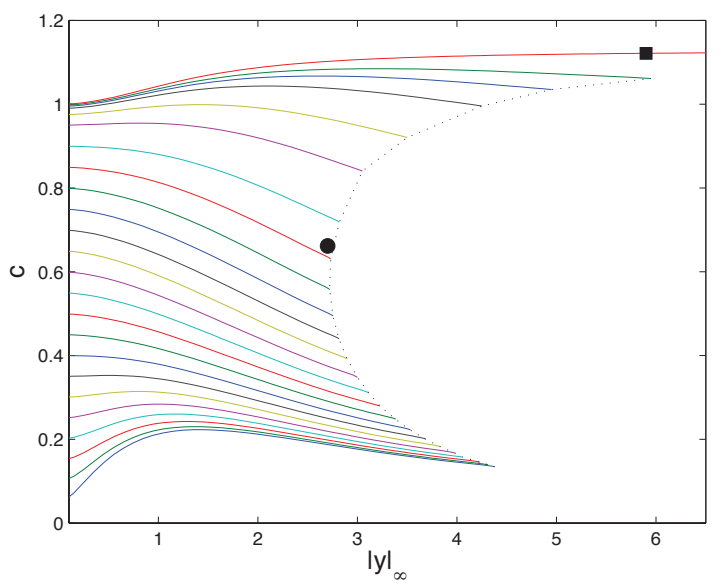

FIG. 1. The speed of computed traveling waves is plotted as a function of the maximum interface displacement, $|y|_{\infty}$. Numerical continuation was used to compute branches of traveling waves for a range of $\gamma_{0} \in[0,2)$. The lowest curve has $\gamma_{0}=1.9975$, while the top curve has $\gamma_{0}=0$. The waves whose profiles appear in Figure 2 are marked with a solid square $\left(\gamma_{0}=0\right)$ and solid circle $\left(\gamma_{0}=1\right)$. The amplitude at which the waves self-intersect is estimated with the dotted curve. We do not observe self-intersection for $\gamma_{0}=0$.

We search for symmetric solutions, where the free surface $y(\alpha, 0)$ is a real even function, which implies that $a_{n}$ are pure imaginary and $b_{n}$ are real, as well as $a_{-n}=-a_{n}$ and $b_{-n}=b_{n}$. In addition we consider waves where the tangent angle has zero mean and the mean of the vortex sheet strength is specified, $\gamma_{0}$. Thus to compute a traveling wave, we must determine $N$ Fourier coefficients and the speed $c$. We impose that the projection of (12) onto the same wavenumbers must vanish. When $\theta$ is odd and $\gamma$ is even, then $\gamma_{t}$ is odd, thus the projection of $\gamma_{t}=0$ into Fourier space gives $N / 2$ coefficients which should vanish (the Fourier coefficients satisfy the same symmetry as $\theta$ ). The complementary equation, $U=\mathbf{W} \cdot \hat{\mathbf{n}}$, is also real and odd, resulting in another $N / 2$ equations. We close the system with an equation which specifies the solution amplitude.

The computation of the projection of (12) into Fourier space is straightforward, save perhaps the computation of the Birkhoff-Rott integral,

$$
\Phi(\mathbf{W})^{*}=\frac{1}{4 \pi i} P V \int_{0}^{2 \pi} \gamma(\beta) \cot \left(\frac{1}{2}(z(\alpha)-z(\beta))\right) d \beta .
$$

The integral is split into a Hilbert transform, which is computed using its definition in Fourier space as $\widehat{H(f)}=-i \operatorname{sgn}(k) \hat{f}(k)$, and the remainder

$$
\begin{aligned}
\Phi(\mathbf{W})^{*}-\frac{1}{2} H & \left(\frac{\gamma}{z_{\alpha}}\right) \\
& =\frac{1}{4 \pi i} P V \int_{0}^{2 \pi} \gamma(\beta)\left[\cot \left(\frac{1}{2}(z(\alpha)-z(\beta))\right)-\frac{1}{z_{\alpha}(\beta)} \cot \left(\frac{1}{2}(\alpha-\beta)\right)\right] d \beta .
\end{aligned}
$$

The remainder integral, $\Phi(\mathbf{W})^{*}-\frac{1}{2} H\left(\frac{\gamma}{z_{\alpha}}\right)$, is computed using the trapezoidal rule at alternating 


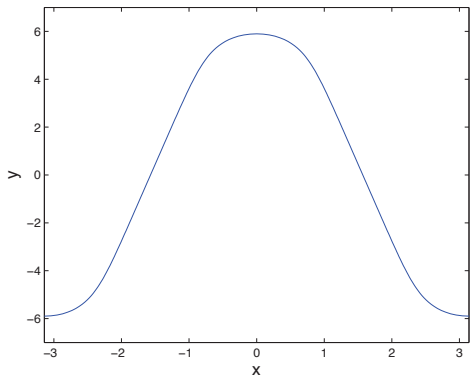

(a) Free Surface, $\gamma_{0}=0$

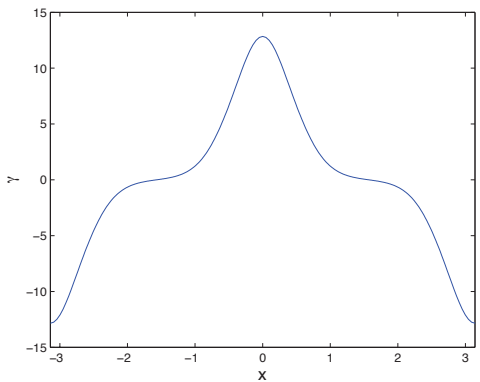

(c) Vortex Sheet Strength, $\gamma_{0}=0$

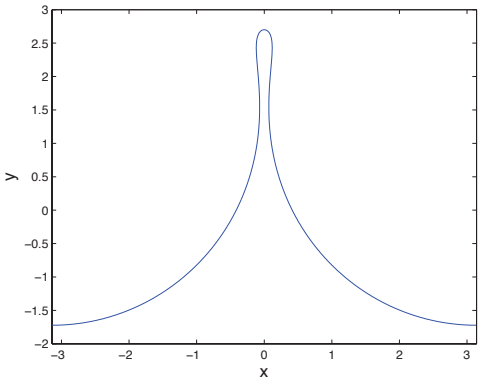

(b) Free Surface, $\gamma_{0}=1$

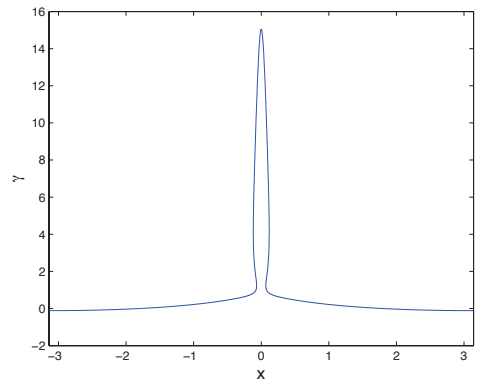

(d) Vortex Sheet Strength, $\gamma_{0}=1$

FIG. 2. The free surface displacement and vortex sheet strength of traveling waves at large amplitude. In the left column, the wave has zero mean vortex sheet strength, $\gamma_{0}=0$. This wave is marked with a solid square in Figure 1 . In the right column, the wave has $\gamma_{0}=1$; this wave is marked with a solid circle in Figure 1 . Both waves were computed with $N=2048$ Fourier modes, with a spatial resolution of $d x=\frac{\pi}{1024} \approx 0.003$. Note that the vortex sheet strength is plotted here as a (possibly multi-valued) function of $x$, rather than as a function of $\alpha$.

grid points, similarly to the procedure of [11]. In the computations presented here, the free surface height at $x=0$ is used to specify amplitude. It is by varying this value that branches of waves are computed. To solve the resulting algebraic system, the quasi-Newton iteration of Broyden is applied [18]. We begin at small amplitude, using the linear solution as an initial guess for fixed surface tension and vortex sheet strength, computing branches of waves via continuation in amplitude. Similar quasi-Newton-Fourier-Continuation schemes have been applied to compute gravity-capillary waves in [4, 6, 54].

For fixed surface tension and vortex sheet strengths, linear solutions travel at speeds

$$
c^{2}=\left(\frac{\tau}{2}|k|-\frac{1}{4} \gamma_{0}^{2}\right)
$$

In our computations we set $\tau=2$ and compute solutions which bifurcate from $k=1$. With these choices, small amplitude traveling waves exist for $\left|\gamma_{0}\right|<2$ and $|c|<1$. The speed-amplitude curves for varying $\gamma_{0}$ are presented in Figure 1 . Worthy of note in Figure 1 is that many of the 

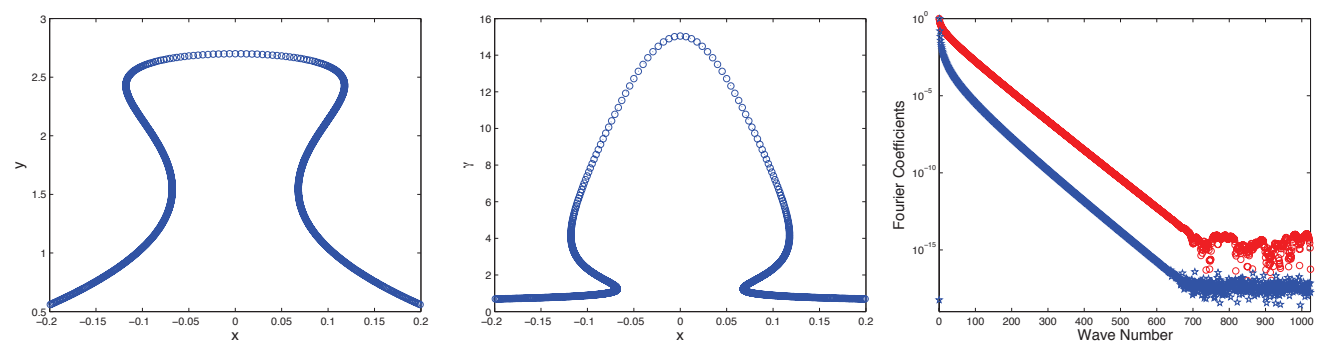

FIG. 3. The overturned traveling wave with $\gamma_{0}=1$, whose entire profile is in the right column of Figure 2 and which is marked with a solid circle in Figure 1. Close-ups of the overturned interface and vortex sheet strength are in the left and center panel respectively, with grid points labeled with circles. Note that the vortex sheet strength is graphed as a multivalued function of $x$, rather than as a function of $\alpha$. The amplitude of the Fourier coefficients is plotted in the right panel as a check of solution smoothness, where the Fourier coefficients of the vortex sheet strength are marked with circles (the upper curve) and the Fourier coefficients for the free surface displacement are marked with stars (the lower curve).

speed-amplitude curves exhibit local maxima, hinting that the stability of these traveling waves may change as a function of amplitude, [3,36]. The spectral stability of such solutions will be considered in a future paper, both to observe potential instabilities of these waves and as a starting point for computations of nontrivially time periodic solutions using the method of [11].

Branches of traveling waves have been computed for a sampling of mean vortex sheet strengths. When $\gamma_{0}=0$, we do not observe overturning traveling waves. A large amplitude solution for $\gamma_{0}=0$ is in the left column of Figure 2. For all sampled values of $\gamma_{0} \neq 0$, overturning solutions were computed at finite amplitude. An example of a large amplitude, overturned traveling wave with $\gamma_{0}=1$ is shown in the right column of Figure 2.

The curvature of the traveling wave profiles tends to increase with amplitude. As such it is natural to be concerned regarding the smoothness of these solutions and the degree to which the computations are resolved. As evidence of the resolution of the computations, close-ups of the overturned section of a traveling solution are plotted in Figure 3 with the grid points labeled. In addition, in the right panel of Figure 3, the Fourier coefficients of the solution are plotted, and these are exponentially decaying until machine precision errors wash out the computation near $10^{-15}$.

In this section we have presented numerical computations of the traveling waves which bifurcate from the linear solution. At large amplitude, many of the solutions overturn. We are able to compute continuous solution branches up to the point where the solution self intersects, so that the wave profile then includes an entrained bubble. In Figure 1, the amplitude where this pinch-off occurs is estimated by the dotted line. The amplitude at which the wave self intersects is a simple estimate of the largest traveling wave, as in [46]. Beyond this threshold the bifurcation structure of the solution space is unclear - and may include multiply intersecting profiles, such as those in [55], as well as regions where no traveling solutions exist.

Acknowledgements. DMA gratefully acknowledges support from the National Science Foundation through grants DMS-1008387 and DMS-1016267. JDW gratefully acknowledges support from the National Science Foundation through grants DMS-0908299 and DMS-1105635. 


\section{REFERENCES}

1. Ablowitz, M., \& Fokas, A. Complex variables: introduction and applications, second ed. Cambridge Texts in Applied Mathematics. Cambridge University Press, Cambridge, 2003. Zbl1088. 30001 MR1989049

2. Akers, B., \& Milewski, P. Model equations for gravity-capillary waves in deep water. Studies in Applied Mathematics 121 (2008), 49-69. Zbl1191. 35211 MR2428544

3. Akers, B., \& MilewsKi, P. A stability result for solitary waves in nonlinear dispersive equations. Commun. Math. Sci. 6 (2008), 791-797. Zbl1149.76022 MR2455477

4. Akers, B., \& MiLEWSKi, P. A model equation for wavepacket solitary waves arising from capillarygravity flows. Studies in Applied Mathematics 122 (2009), 249-274. Zb11173. 35114 MR2504629

5. Akers, B., \& Milewski, P. Dynamics of three-dimensional gravity-capillary solitary waves in deep water. SIAM Journal on Applied Mathematics 70 (2010), 2390-2408. Zb105876544 MR2678044

6. Akers, B., \& Nicholls, D. Traveling waves in deep water with gravity and surface tension. SIAM Journal on Applied Mathematics 70 (2010), 2373-2389. Zb105876543 MR2678043

7. Akers, B., \& Nicholls, D. Spectral stability of deep two-dimensional gravity water waves: repeated eigenvalues. SIAM J. Appl. Math. 72 (2012), 689-711. Zbl06064788 MR2914345

8. Ambrose, D. Well-posedness of vortex sheets with surface tension. SIAM J. Math. Anal. 35 (2003), 211-244 (electronic). Zbl1107.76010 MR2001473

9. Ambrose, D., Siegel, M., \& Tlupova, S. A small scale decomposition for 3D boundary integral computations with surface tension. J. Comp. Phys. (2013). Accepted.

10. Ambrose, D., \& Wilkening, J. Global paths of time-periodic solutions of the Benjamin-Ono equation connecting pairs of traveling waves. Commun. Appl. Math. Comput. Sci. 4 (2009), 177-215. Zbl1184. 35271 MR2565874

11. Ambrose, D., \& Wilkening, J. Computation of symmetric, time-periodic solutions of the vortex sheet with surface tension. Proceedings of the National Academy of Sciences 107 (2010), 3361-3366.

12. Ambrose, D., \& Wilkening, J. Computation of time-periodic solutions of the Benjamin-Ono equation. J. Nonlinear Sci. 20 (2010), 277-308. Zbl1203. 37085 MR2639896

13. Amick, C., Fraenkel, L., \& Toland, J. On the Stokes conjecture for the wave of extreme form. Acta Math. 148 (1982), 193-214. Zb10495.76021 MR0666110

14. Amick, C., \& TURner, R. A global theory of internal solitary waves in two-fluid systems. Trans. Amer. Math. Soc. 298 (1986), 431-484. Zbl0631. 35029 MR0860375

15. Ascher, U., RuUth, S., \& Wetton, B. Implicit-explicit methods for time-dependent partial differential equations. SIAM J. Numer. Anal. 32 (1995), 797-823. Zb10841.65081 MR1335656

16. BAKer, G., Meiron, D., \& OrszaG, S. Generalized vortex methods for free-surface flow problems. $J$. Fluid Mech. 123 (1982), 477-501. Zbl0507.76028 MR0687014

17. Beale, J. The existence of solitary water waves. Comm. Pure Appl. Math. 30 (1977), 373-389. Zblo379. 35055 MR0445136

18. Broyden, C. A class of methods for solving nonlinear simultaneous equations. Math. Comp. 19 (1965), 577-593. Zb10131.13905 MR0198670

19. Constantin, A., \& Strauss, W. Exact steady periodic water waves with vorticity. Comm. Pure Appl. Math. 57 (2004), 481-527. Zbl1038.76011 MR2027299

20. Craig, W., \& Nicholls, D. Travelling two and three dimensional capillary gravity water waves. SIAM J. Math. Anal. 32 (2000), 323-359. Zbl0976. 35049 MR1781220

21. Craig, W., \& Nicholls, D. Traveling gravity water waves in two and three dimensions. Eur. J. Mech. B Fluids 21 (2002), 615-641. Zbl1084.76509 MR1947187

22. Crandall, M., \& Rabinowitz, P. Bifurcation from simple eigenvalues. J. Functional Analysis 8 (1971), 321-340. Zb10219.46015 MR0288640 
23. Crapper, G. An exact solution for progressive capillary waves of arbitrary amplitude. J. Fluid Mech. 2 (1957), 532-540. Zbl0079.19304 MR0091075

24. CRAPPER, G. Introduction to water waves. Ellis Horwood Series: Mathematics and its Applications. Ellis Horwood Ltd., Chichester, 1984. Zbl0546.76029 MR0769846

25. Dias, F., \& Iooss, G. Capillary-gravity interfacial waves in infinite depth. European J. Mech. B Fluids 15 (1996), 367-393. Zb10887. 76014 MR1400516

26. Friedrichs, K., \& Hyers, D. The existence of solitary waves. Comm. Pure Appl. Math. 7 (1954), 517-550. Zbl0057.42204 MR0065317

27. Grimshaw, R., \& Pullin, D. Extreme interfacial waves. Phys. Fluids 29 (1986), 2802-2807.

28. GRoves, M., \& SUN, S.-M. Fully localised solitary-wave solutions of the three-dimensional gravitycapillary water-wave problem. Arch. Ration. Mech. Anal. 188 (2008), 1-91. Zbl1133. 76010 MR2379653

29. Hamilton, J., Kim, J., \& WalefFe, F. Regeneration mechanisms of near-wall turbulence structures. Journal of Fluid Mechanics 287 (1995), 317-348. Zb10867. 76032

30. Helson, H. Harmonic analysis. Addison-Wesley Publishing Company Advanced Book Program, Reading, MA, 1983. Zbl0555.43001 MR0729682

31. Hou, T., Lowengrub, J., \& Shelley, M. Removing the stiffness from interfacial flows with surface tension. J. Comput. Phys. 114 (1994), 312-338. Zbl0810.76095 MR1294935

32. Hou, T., Lowengrub, J., \& Shelley, M. The long-time motion of vortex sheets with surface tension. Phys. Fluids 9 (1997), 1933-1954. Zbl1185.76545 MR1455083

33. Iooss, G. Gravity and capillary-gravity periodic travelling waves for two superposed fluid layers, one being of infinite depth. J. Math. Fluid Mech. 1 (1999), 24-61. Zbl0926.76020 MR1699018

34. Kawahara, G., \& Kida, S. Periodic motion embedded in plane Couette turbulence: regeneration cycle and burst. Journal of Fluid Mechanics 449 (2001), 291-300. Zbl0996 . 76034 MR1871646

35. Levi-Civita, T. Determinazione rigorosa delle onde irrotazionali periodiche in acqua profonda. Rend. Accad. Lincei. 33 (1924), 141-150.

36. Mackay, R., \& Saffman, P. Stability of water waves. Proc. Roy. Soc. London Ser. A 406 (1986), 115-125. Zbl0602.76046 MR0853684

37. MCLeod, J. B. The Stokes and Krasovskii conjectures for the wave of greatest height. Stud. Appl. Math. 98 (1997), 311-333. Zbl0882.76014 MR1446239

38. Meiron, D., \& Saffman, P. Overhanging interfacial gravity waves of large amplitude. J. Fluid. Mech. 129 (1983), 213-218. Zbl0516.76019

39. Nekrasov, A. On steady waves. Izv. Ivanovo-Voznesenk. Politekhn. 3 (1921).

40. Nicholls, D. Spectral data for travelling water waves: singularities and stability. J. Fluid Mech. 624 (2009), 339-360. MR2496379

41. Окамото, H. Uniqueness of Crapper's pure capillary waves of permanent shape. J. Math. Sci. Univ. Tokyo 12 (2005), 67-75. Zb11078.76016 MR2126785

42. Окамото, H., \& Sно̄JI, M. The mathematical theory of permanent progressive water-waves, vol. 20 of Advanced Series in Nonlinear Dynamics. World Scientific Publishing Co. Inc., River Edge, NJ, 2001. Zb10983.76002 MR1869386

43. Plotnikov, P. Proof of the Stokes conjecture in the theory of surface waves. Stud. Appl. Math. 108 (2002), 217-244. Translated from Dinamika Sploshn. Sredy No. 57 (1982), 41-76. Zb10522.76017 MR1883094

44. SAFFMAN, P., \& YUEN, H. Finite-amplitude interfacial waves in the presence of a current. J. Fluid Mech. 123 (1982), 459-476. Zbl0502.76026 MR0687013

45. Saffman, P. G. Vortex dynamics. Cambridge Monographs on Mechanics and Applied Mathematics. Cambridge University Press, New York, 1992. Zb10777.76004 MR1217252

46. Schwartz, L., \& VAnden-Broeck, J.-M. Numerical solution of the exact equations for capillary- 
gravity waves. Journal of Fluid Mechanics 9501 (1979), 119-139. Zb10419.76014 MR0555210

47. Sun, S.-M. Asymptotic behavior and symmetry of internal waves in two-layer fluids of great depth. $J$. Differential Equations 129 (1996), 18-48. Zbl0859.76012 MR1400795

48. SUN, S.-M. Periodic waves in two-layer fluids of infinite depth. In Advances in multi-fluid flows (Seattle, WA, 1995). SIAM, Philadelphia, PA, 1996, pp. 339-345. Zb10877.76011 MR1414910

49. Sun, S.-M. Some analytical properties of capillary-gravity waves in two-fluid flows of infinite depth. Proc. Roy. Soc. London Ser. A 4531961 (1997), 1153-1175. Zbl0885.76015 MR1455326

50. SUN, S.-M. Existence of large amplitude periodic waves in two-fluid flows of infinite depth. SIAM J. Math. Anal. 32 (2001), 1014-1031 (electronic). Zb10980. 35133 MR1828316

51. Sun, S.-M., \& SHEN, M. Solitary waves in a two-layer fluid with surface tension. SIAM J. Math. Anal. 24 (1993), 866-891. Zb10783.76019 MR1226855

52. Toland, J. On the existence of a wave of greatest height and Stokes's conjecture. Proc. Roy. Soc. London Ser. A 3631715 (1978), 469-485. Zbl0388.76016 MR0513927

53. Turner, R., \& VAnden-Broeck, J.-M. The limiting configuration of interfacial gravity waves. Phys. Fluids 29 (1986), 372-375. MR0828169

54. VANDEN-BRoECK, J.-M., \& DiAs, F. Gravity-capillary solitary waves in water of infinite depth and related free-surface flows. Journal of Fluid Mechanics 240 (1992), 549-557. Zb10775 . 76021 MR1175093

55. VAnden-Broeck, J.-M., \& Keller, J. A new family of capillary waves. Journal of Fluid Mechanics 9801 (1980), 161-169.

56. Viswanath, D. Recurrent motions within plane Couette turbulence. Journal of Fluid Mechanics 580 (2007), 339-358. Zbl1175.76074 MR2326295

57. Wilkening, J. Breakdown of self-similarity at the crests of large-amplitude standing water waves. Phys. Rev. Lett. 107 (Oct 2011), 184501.

58. Wu, S. Well-posedness in Sobolev spaces of the full water wave problem in 2-D. Invent. Math. 130 (1997), 39-72. Zbl0892.76009 MR1471885

59. ZEIDLER, E. Beiträge zur Theorie und Praxis freier Randwertaufgaben. Funktionalanalytische Untersuchungen über eine Klasse nichtlinearer hydrodynamischer Probleme. Akademie-Verlag, Berlin, 1971. Schriftenreihe der Institute für Mathematik bei der Deutschen Akademie der Wissenschaften zu Berlin, Reihe A. Reine Mathematik, No. 9. Zb10232. 35003 MR0349136

60. ZEIDLER, E. Applied functional analysis, vol. 109 of Applied Mathematical Sciences. Springer-Verlag, New York, 1995. Main principles and their applications. Zb10834.46003 MR1347692 